\title{
Estrogen receptors and human disease
}

\author{
Bonnie J. Deroo and Kenneth S. Korach
}

\author{
Receptor Biology Section, National Institute of Environmental Health Sciences, NIH, Research Triangle Park, North Carolina, USA.
}

\begin{abstract}
Estrogens influence many physiological processes in mammals, including but not limited to reproduction, cardiovascular health, bone integrity, cognition, and behavior. Given this widespread role for estrogen in human physiology, it is not surprising that estrogen is also implicated in the development or progression of numerous diseases, which include but are not limited to various types of cancer (breast, ovarian, colorectal, prostate, endometrial), osteoporosis, neurodegenerative diseases, cardiovascular disease, insulin resistance, lupus erythematosus, endometriosis, and obesity. In many of these diseases, estrogen mediates its effects through the estrogen receptor (ER), which serves as the basis for many therapeutic interventions. This Review will describe diseases in which estrogen, through the ER, plays a role in the development or severity of disease.
\end{abstract}

\section{Estrogen receptor mechanism of action}

Estrogens induce cellular changes through several different mechanisms (Figure 1). Central to these mechanisms is the protein to which estrogens bind, the estrogen receptor (ER). In the "classical" mechanism of estrogen action, estrogens diffuse into the cell and bind to the ER, which is located in the nucleus. This nuclear estrogen-ER complex binds to estrogen response element sequences directly or indirectly through protein-protein interactions with activator protein 1 (AP1) or SP1 sites in the promoter region of estrogen-responsive genes, resulting in recruitment of coregulatory proteins (coactivators or corepressors) to the promoter, increased or decreased mRNA levels and associated protein production, and a physiological response. This classical, or "genomic," mechanism typically occurs over the course of hours. In contrast, estrogen can act more quickly (within seconds or minutes) via "nongenomic" mechanisms, either through the ER located in or adjacent to the plasma membrane, or through other non-ER plasma membraneassociated estrogen-binding proteins (Figure 1), resulting in cellular responses such as increased levels of $\mathrm{Ca}^{2+}$ or $\mathrm{NO}$, and activation of kinases. The ER may be targeted to the plasma membrane by adaptor proteins such as caveolin-1 or Shc.

The ER exists in 2 main forms, ER $\alpha$ and $E R \beta$, which have distinct tissue expression patterns (1) in both humans and rodents, and gene-targeted animal models lacking these receptors exhibit distinct phenotypes and provide some of the most definitive experimental models for evaluating the role of the ER in disease and normal physiology (2). ER $\alpha$ and ER $\beta$ are encoded by separate genes, ESR1 and ESR2, respectively, found at different chromosomal locations, and numerous mRNA splice variants exist for both receptors in both diseased and normal tissue (Figure 2), although the corresponding protein products have been difficult to confirm (3). Because these splice variants are frequently coexpressed with their wild-type counterparts, the exact function and potential role of these variants in normal physiology and human disease remain to be elucidated.

Nonstandard abbreviations used: $\mathrm{AD}$, Alzheimer disease; $\mathrm{BMD}$, bone mineral density; DA, dopamine; DES, diethylstilbestrol; ER, estrogen receptor; $\alpha E R K O$, ER $\alpha$ knockout; $\beta E R K O, E R \beta$ knockout; FL, femur length; HRT, hormone replacement therapy; LH, luteinizing hormone; LH $\beta C T$, LH $\beta$-C-terminal peptide; LPL, lipoprotein lipase; PAX2, paired-box gene 2; PD, Parkinson disease; SERM, selective estrogen receptor modulator; SNP, single-nucleotide polymorphism; WHI,

Women's Health Initiative.

Conflict of interest: The authors have declared that no conflict of interest exists. Citation for this article: J. Clin. Invest. 116:561-570 (2006). doi:10.1172/JCI27987.
Selective estrogen receptor modulators (SERMs) such as tamoxifen and raloxifene (Figure 3) are examples of compounds that exhibit tissue-specific estrogenic activity. Tamoxifen, although an ER agonist in bone and uterus, is an antagonist in the breast and has been a safe and effective adjuvant endocrine therapy for breast cancer for almost 20 years. Raloxifene is similar to tamoxifen in its tissue-specific agonist/antagonist profile but exhibits greater agonist activity in bone and less in the uterus (4) - hence its use for the prevention of osteoporosis (see "ERs and osteoporosis," below). Whether a SERM is an ER agonist or antagonist in a particular tissue depends on several factors. Binding of a SERM to the ER causes a specific conformational change in the receptor, and the resulting 3D structure determines which coactivators and/or corepressors are recruited to the promoter. Thus, the relative level of corepressors and coactivators in a particular tissue is a critical determinant of a SERM's agonist/antagonist activity. For example, tamoxifen recruits a coactivator complex to estrogen-regulated genes in endometrial cells but a corepressor complex to the same gene in breast cancer cells (5). The specific coregulators recruited to a particular promoter also depend on the type of ER-dependent regulatory sequences that are present in the promoter, such as estrogen response elements or other transcription factor binding sites, such as AP1. Lastly, the $\mathrm{ER} \alpha / \mathrm{ER} \beta$ ratio varies between tissues, and which ER form predominates also affects SERM activity. Thus, the physiological response to a particular SERM results from a combination of factors, including its chemical structure and the cellular and promoter context in which the SERM acts.

In parallel with the development of therapeutic strategies based on the modulation of ER activity, compounds that inhibit the production of estrogen have also been generated and are currently in use clinically. Inhibitors of aromatase, the enzyme that converts androgens to estrogens, have been successfully used in the treatment of breast cancer and other diseases. Thus, targeting both the ER and the production of its activating ligand provides complementary strategies for the management of estrogen-regulated diseases.

\section{ERs and cancer}

Breast cancer. Estrogen is implicated in the development of breast cancer, based on data from both clinical and animal studies; risk factors associated with breast cancer reflect cumulative exposure of the breast epithelium to estrogen (6). Two current hypotheses exist to explain this relationship (7). In the first, binding of estrogens to the ER stimulates proliferation of mammary cells, increasing the target cell number within the tissue, and the increase in cell 


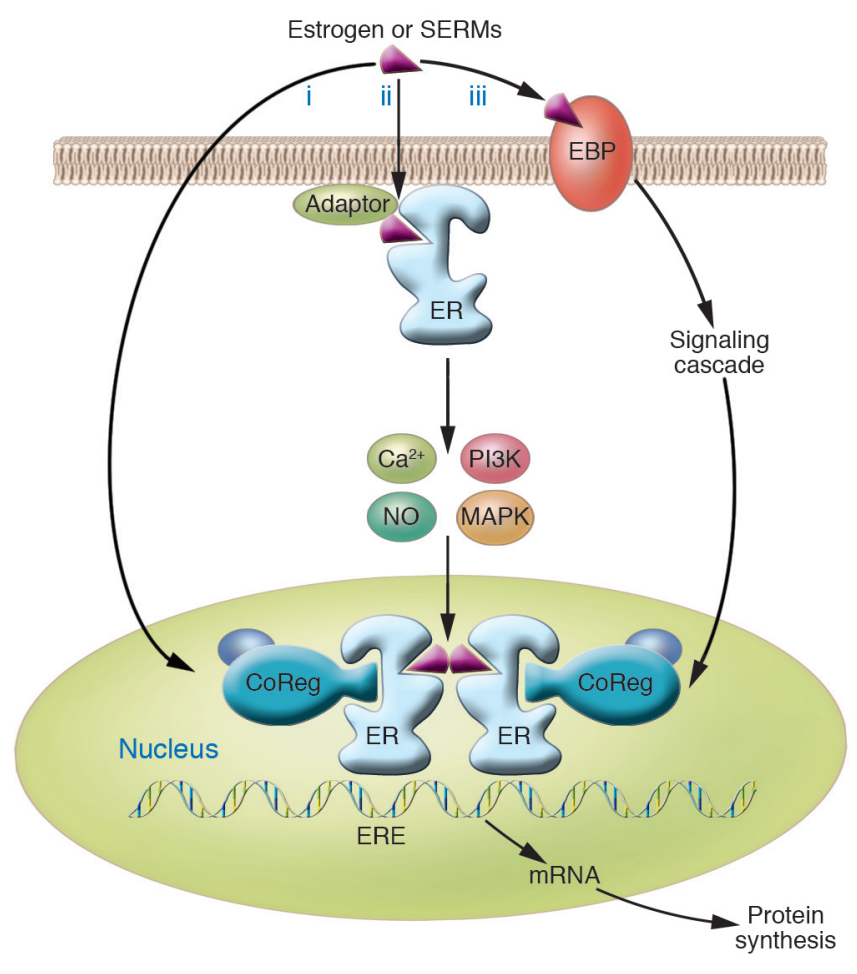

division and DNA synthesis elevates the risk for replication errors, which may result in the acquisition of detrimental mutations that disrupt normal cellular processes such as apoptosis, cellular proliferation, or DNA repair. In the second hypothesis, estrogen metabolism leads to the production of genotoxic by-products that could directly damage DNA, again resulting in point mutations. There is evidence that estrogen may act through both mechanisms to initiate and/or promote mammary cancer.

Currently, both SERMs and aromatase inhibitors are used in the treatment of breast cancer, and patients whose tumors are ER-positive respond well to these endocrine therapies. Further, both tamoxifen and raloxifene have proven effective as chemopreventative agents for breast cancer in high-risk women (4). The randomized, clinical Raloxifene Use for the Heart (RUTH) trial is currently testing the efficacy of chronic raloxifene treatment in postmenopausal women in (a) reducing the incidence of coronary heart disease (multiple endpoints), and (b) reducing the risk of invasive breast cancer in women at high risk for major coronary events (8). In addition, ICI 182,780 (known clinically as Faslodex), an ER antagonist that inhibits ER activity and reduces ER levels, is also used in the treatment of breast cancer. ER $\alpha$ expression is associated with more differentiated tumors and a more favorable prognosis, and although the potential role of ER $\beta$ in tumor progression is controversial, studies of ER $\beta$ protein levels suggest that it is also a favorable prognostic indicator (3).

Several sequence variations or single-nucleotide polymorphisms (SNPs) in the ER $\alpha$ gene (ESR1) have been identified that are associated with either an increased or a decreased risk of breast cancer. The best-characterized SNPs of ESR1 are the PvuII and XbaI restriction site polymorphisms, both of which are located in the first intron $(9,10)$. The PvuII polymorphism (also known as ESR1 c. $454-397 \mathrm{~T} \rightarrow \mathrm{C}$ ) is associated with increased breast cancer risk (11), as well as risk for other diseases in which estrogen is impli-

\section{Figure 1}

Models of estrogen action. In the "classical" pathway of estrogen action (i), estrogen or other selective estrogen receptor modulators (SERMs) bind to the estrogen receptor (ER), a ligand-activated transcription factor that regulates transcription of target genes in the nucleus by binding to estrogen response element (ERE) regulatory sequences in target genes and recruiting coregulatory proteins (CoRegs) such as coactivators. Rapid or "nongenomic" effects of estrogen may also occur through the ER located in or adjacent to the plasma membrane (ii), which may require the presence of "adaptor" proteins, which target the ER to the membrane. Activation of the membrane ER leads to a rapid change in cellular signaling molecules and stimulation of kinase activity, which in turn may affect transcription. Lastly, other non-ER membrane-associated estrogen-binding proteins (EBPs) may also trigger an intracellular response (iii).

cated. SNPs in the gene for ER $\beta$ (ESR2) have also been identified, but their association with breast cancer risk is less clear than that of SNPs in ESR1. Zheng et al. identified 2 ESR2 SNPs associated with increased breast cancer risk in postmenopausal but not in premenopausal women (12). A recent study of 3 ESR2 SNPs found no association of the individual SNPs, but 1 haplotype of these SNPs was identified that was associated with an increased risk of sporadic breast cancer (13). Further analysis of the functional consequences of SNPs for ESR1 and ESR2 and association of SNPs in particular subject study groups, as well as identification of novel SNPs, may shed further light on the hereditary contributions to the development of breast cancer.

Both cell culture and animal model studies indicate that the ER is involved in mammary gland development and mammary cancer. Studies in ER $\alpha$ knockout ( $\alpha \mathrm{ERKO})$ mice demonstrate that ER $\alpha$ is required for normal mammary gland development (14). Adult $\alpha$ ERKO females exhibit mammary glands that are similar to those of normal prepubertal female mice, i.e., lacking ductal development and terminal end buds. In Neu/ErbB2 knock-in transgenic mice, which develop mammary tumors with a long latency period, loss of ER $\alpha$ resulted in no incidence of tumor development, indicating that ER $\alpha$ contributes to the development and/or progression of Neu-induced carcinogenesis in this model (V. Giguère, personal communication). However, in a second Neu model, $\alpha$ ERKO animals were crossed with mice expressing a mammary-specific mutant Neu, which was constitutively active (resulting in much higher levels of the oncogene compared with the first model). In this second model, females that lacked ER $\alpha$ still developed mammary tumors, although tumor onset was delayed (15). These studies indicate that ER $\alpha$ influences tumor progression but not incidence for $\mathrm{Nen}$-induced carcinogenesis when the oncogene is highly expressed. In the $\mathrm{C} 3(1) / \mathrm{T}(\mathrm{AG})$ mouse model of estrogen-promoted mammary tumorigenesis, mammary tumor incidence is minimized with the loss of ER $\alpha$ expression (16). These data suggest that ERs may serve different functions, depending on the stage of tumor development and/or progression. Thus, both basic science and clinical data indicate that ER $\alpha$ is a primary mediator of estrogenic actions in breast cancer.

These ER-dependent actions of estrogen in breast cancer also involve transcriptional coregulators, such as members of the SRC family (17). For example, the coactivator amplified in breast cancer-1 (AIB1, also known as SRC3), which is overexpressed in some breast tumors, mediates hormone-dependent cellular proliferation in cultured cells, and tumor initiation and growth in mouse models of mammary tumorigenesis (18). The mechanisms by which ERs and coregulators regulate tumor development and progres- 
A
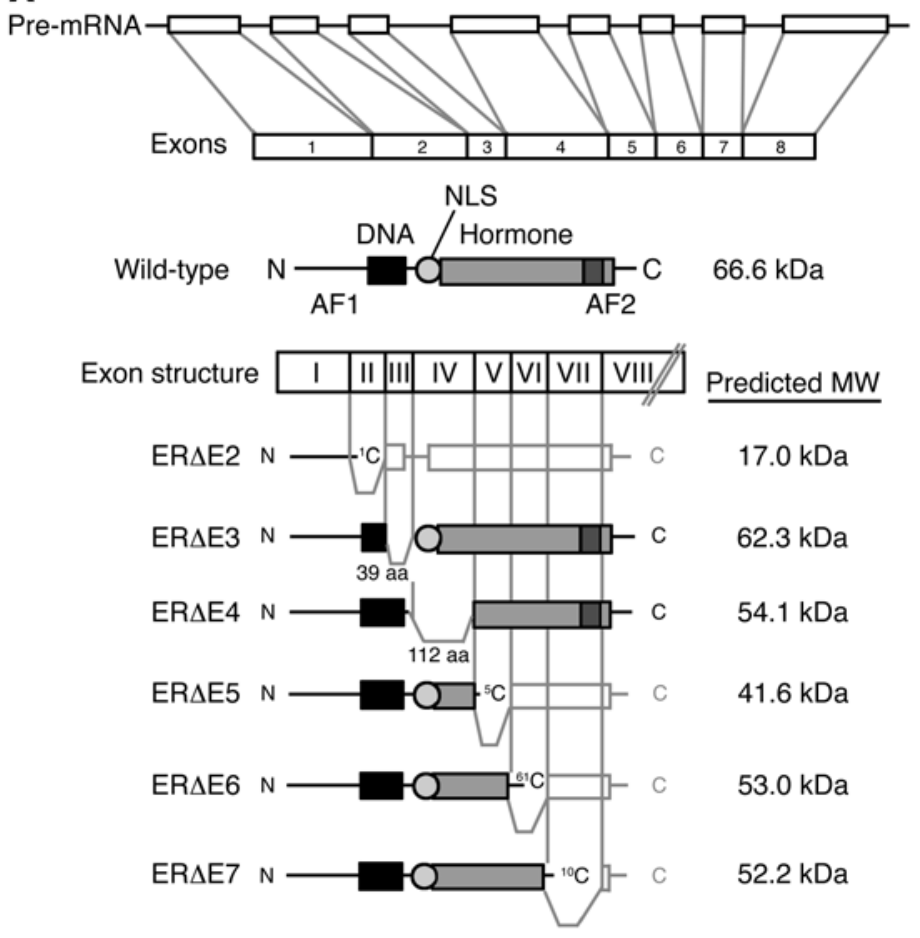

$53.0 \mathrm{kDa}$

$52.2 \mathrm{kDa}$
B

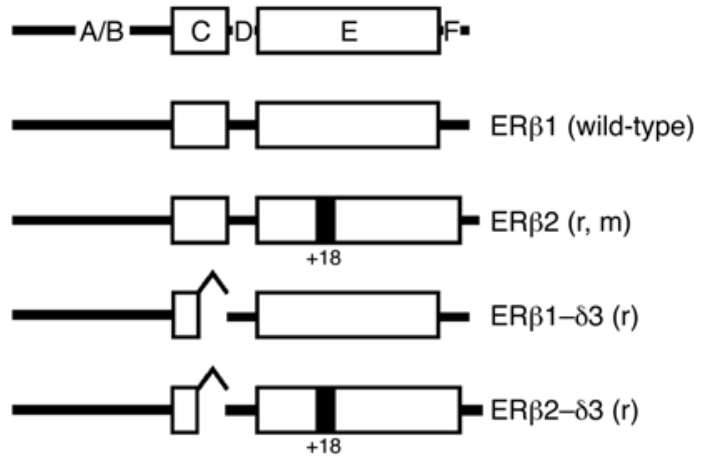

$17.0 \mathrm{kDa}$

$62.3 \mathrm{kDa}$

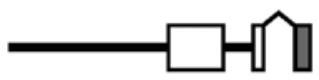

$\operatorname{ER} \beta 1-\delta 5(h, m, b, o, p)$

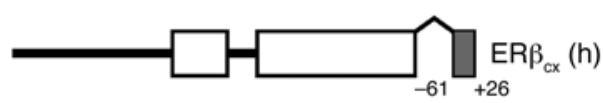

$\square \prod_{-61} \operatorname{ER} \beta 4(h)$

\section{Figure 2}

Exon structure, primary transcript, and common mRNA splice variants of the ER. (A) The most common splice variants of ER $\alpha$ are expressed in multiple tissues and arise from deletions of internal exons, resulting in truncated proteins lacking segments of the DNA-binding domain (DBD) or hormone-binding (ligand-binding) domain (LBD) of the receptor. Most variant isoforms possess little transcriptional activity, with the exception of $\mathrm{ER} \triangle \mathrm{E} 5$, which binds DNA but lacks most of the LBD, resulting in low levels of constitutive activity in some cell lines. ER $\triangle E 2$ lacks the DBD and the dimerization domain, while ER $\triangle \mathrm{E} 3$ lacks part of the DBD. ER $\Delta \mathrm{E} 4$, which lacks part of the LBD, does not bind DNA or hormone, and ER $\triangle \mathrm{E} 6$ is missing part of the LBD and dimerization domain. ER $\Delta E 7$ lacks the activation function 2 (AF2) domain and part of the LBD. ER $\triangle E 3$, ER $\Delta E 5$, and ERAE7 variants have demonstrated a dominant-negative effect on transcriptional activity mediated by wild-type ER. Adapted with permission from Molecular Endocrinology (S33). (B) Mammalian ER $\beta$ variants identified in humans ( $h$ ), rats ( $r$ ), mice ( $m$ ), cows (b), sheep (o), and pigs (p). As described for $E R \alpha$, wild-type ER $\beta$ (ER $\beta 1$ ) possesses both a DBD (C domain) and an LBD (E domain). ER $\beta 2$ codes for a variant that contains an additional 18 amino acids in the LBD, while ER $\beta 1-\delta 3$ lacks exon 3 and therefore part of the DBD. ER $\beta 2-\delta 3$ contains both of these variations. $E R \beta 1-\delta 5$ lacks exon 5 , and in $E R \beta_{C x}$, the $C$-terminal 61 amino acids are replaced by a unique sequence of 26 amino acids. ER $\beta 4$ is truncated at both the $\mathrm{N}$ and the $\mathrm{C}$ termini. In humans, variants lacking exon 2, exon 4 , exon 6 , and exon 7 also exist. Adapted from ref. S34.

sion are now beginning to be understood, and future research will increase our understanding of the variety of coregulators involved and the mechanisms by which they regulate estrogen-dependent transcription and cellular growth.

Ovarian cancer. In the US, ovarian cancer is the fifth most common cancer in females (19). Eighty to ninety percent of ovarian tumors arise from the ovarian surface epithelium, while approximately $5 \%$ are derived from granulosa cells. There is evidence that both steroid hormones and gonadotropins contribute to the etiology of ovarian cancer in humans. The ER is present in approximately two-thirds of human ovarian tumors, with ER $\alpha$ being expressed in tumors of both epithelial and stromal origin, whereas ER $\beta$ is abundantly expressed predominantly in granulosa cell-derived tumors and to a lesser extent in mucinous tumors of epithelial origin (20). A role for ERs in mediating ovarian cancer cell growth is supported by cell culture studies. Estrogens stimulate proliferation of ovarian cancer cell lines and normal ovarian surface epithelial cells in culture (21, 22). In contrast, growth is inhibited by ER antagonists (21). Despite these findings, clinical trials indicate that tamoxifen does not effectively inhibit recurrence of ovarian cancers $(23,24)$.
Gonadotropin stimulation is also implicated in the etiology of ovarian cancer, since gonadotropins stimulate steroidogenesis and increase localized estrogen levels in the ovary, and ERs may be involved in this process. In rodents, disruption of negative feedback on the hypothalamic-pituitary axis elevates gonadotropin levels, and this chronic stimulation leads to development of ovarian tumors. Experimental animals with elevated luteinizing hormone $(\mathrm{LH})$ levels, specifically $\alpha$ ERKO mice and transgenic LH $\beta$-C-terminal peptide (LHßCTP) mice, develop interstitial/stromal hyperplasia and granulosa cell-derived tumors $(2,25)$. Interestingly, ER $\beta$ appears to be involved in the manifestation of both interstitial and granulosa cell hyperplasia in LH $\beta$ CTP mice, since the incidence of luteinized interstitial hyperplasia and granulosa cell hyperplasia was reduced in LH $\beta C$ TP mice lacking ER $\beta$ (26). However, granulosa cell tumors develop in inhibin- $\alpha$-null (Inha-/-) mice lacking ER $\alpha$ or ER $\beta$, suggesting that ER signaling is not required for granulosa cell tumor development in this model (27). Thus, the role of estrogen in ovarian cancer remains to be fully understood.

Colon cancer. ER $\beta$ is the predominant ER form in both normal and malignant human colon tissue, as well as several human colon 


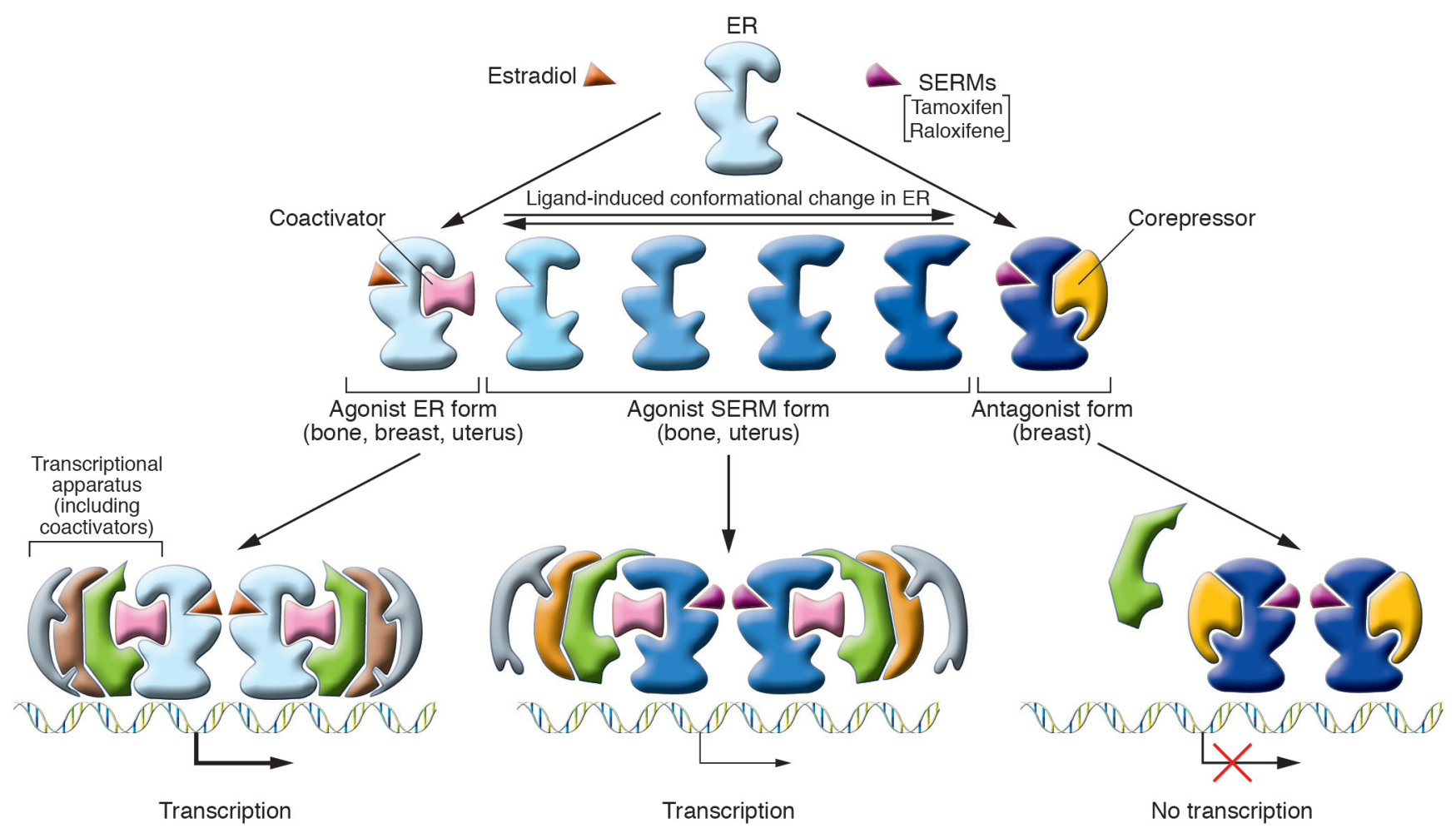

Figure 3

Differential ER structure and coactivator recruitment by ER agonists, antagonists, and SERMs. Upon binding ER ligands such as estradiol or SERMs, the receptor undergoes a conformational change, allowing the ER to exist in a spectrum of conformations from active to inactive depending on the nature of the bound ligand. This conformation, in turn, regulates the recruitment of specific transcriptional coregulatory proteins and the resulting transcriptional apparatus. Coactivators such as SRC1 bind to the active (agonist-bound) form of the receptor and activate transcription, while corepressors interact with the antagonist-bound receptor, inhibiting transcription. Depending on the cellular and promoter context, both unique and overlapping sets of genes may be regulated by various ligands. Adapted with permission from The American Journal of Cardiology (S35).

cancer-derived cell lines (28-31). ER $\beta$ protein levels are reportedly lower in colon tumors compared with normal colon tissue, and loss of ER $\beta$ is associated with advanced stages of colon cancer and tumor cell dedifferentiation, suggesting a protective role for ER $\beta$ in colon tumorigenesis $(30,32-34)$. Clinical studies indicate that the incidence of colon cancer is lower in women than in men (35), and data from the Women's Health Initiative (WHI) indicate a significantly reduced incidence of colon cancer in postmenopausal women receiving combined hormone replacement therapy (HRT; estrogen plus progestin) (36), consistent with a protective role of ER $\beta$. Development of an ER $\beta$-specific agonist for treatment of inflammatory bowel disease (i.e., Crohn disease) and rheumatoid arthritis has been reported (37). In vitro, both tamoxifen and raloxifene inhibit proliferation and viability of colon cancer cell lines, which may indicate the value of SERMs for the prevention and treatment of colon cancer (38).

Prostate cancer. Since the late 1980s, prostate cancer has been the second leading cause of death due to cancer in men (35). To date, the clinical evidence linking increased serum estrogen levels or an increased estrogen/androgen ratio with elevated risk of prostate cancer remains inconclusive (39). Still, experimental data in rodents strongly suggest that prolonged or prenatal/neonatal exposure to estrogens can affect prostate development and function and cause epithelial hyperplasia including drastic alterations in the expression pattern of steroid receptors (40). In both human and rodent prostate, $E R \beta$ is more highly expressed than $E R \alpha$, and while $E R \beta$ is found in both epithelial and (to a lesser extent) stromal cells, ER $\alpha$ is generally localized to the stroma (40). Although the role of the ERs in prostate physiology remains unclear, ER $\beta$ expression is reportedly reduced in prostate tumors $(41,42)$, and prostatic epithelial hyperplasia has been reported in adult ER $\beta$ knockout ( $\beta$ ERKO) mice (43). Recent studies have suggested a protective role for ER $\beta$ specific agonists, as treatment of rodents with these compounds results in prostate atrophy due to apoptosis (44).

In rodents, neonatal exposure to $17 \beta$-estradiol (estradiol) or other estrogens such as diethylstilbestrol (DES) disrupts normal morphogenesis and inhibits prostate growth, resulting in aberrant prostate function and morphology in the adult prostate, as well as inflammation, hyperplasia, and dysplasia (45). ER $\alpha$ is the key mediator of these estrogenic effects, since neonatal DES exposure of $\alpha E R K O$ mice did not result in developmental estrogenization of the prostate resulting in prostate hyperplasia (46). A role for ER $\alpha$ in estrogenic actions in the prostate is also supported by the observation that adult $\alpha E R K O$ mice treated with DES do not develop the prostatic squamous metaplasia observed in DES-treated wildtype mice (47). Despite the experimental evidence supporting a role for ERs in prostate development and normal function in rodents, clinical trials with the SERMs tamoxifen and toremifene have demonstrated limited efficacy in inhibition of disease progression of androgen-independent tumors $(48,49)$. 
Endometrial cancer. Approximately $70-80 \%$ of sporadic endometrial carcinomas are distinguished as type I carcinomas and are associated with endometrial hyperplasia, hyperestrogenism, and expression of ER. The remaining $20 \%$ constitute type II carcinomas, are generally unrelated to estrogen, and exhibit negative or low ER expression (50). Estrogen-dependent endometrial tumors are thought to arise because of prolonged exposure to estrogens in the absence of sufficient progesterone (the "unopposed estrogen hypothesis"), and this hypothesis has been supported by many studies (51). Unopposed estrogen can result from obesity, ovarian tumors that secrete estrogen, estrogen therapy (in the absence of progestin), and tamoxifen treatment (agonist activity), among other causes. The reason for increased risk of endometrial cancer due to tamoxifen treatment is not well understood, but a recent study suggests that tamoxifen and estradiol induce overlapping but distinct sets of genes in both cells from type I endometrial carcinoma and normal endometrial epithelial cells (52). The authors identified a gene, paired-box gene 2 $(P A X 2)$, that demonstrated increased expression after estradiol and tamoxifen treatment of endometrial carcinoma cells but not normal cells. ER $\alpha$ was recruited to the $P A X 2$ promoter in response to both treatments. They found that the PAX2 promoter was hypomethylated in the carcinoma samples compared with the hypermethylation observed in normal endometrium, suggesting that PAX2, which is normally silent, is "reactivated" in endometrial cancer. These and future studies will further clarify the role of ER $\alpha$ in the development and/or progression of endometrial carcinoma and may help to identify diagnostic or therapeutic markers.

\section{ERs and osteoporosis}

Estrogens regulate skeletal homeostasis in both men and women. Osteoporosis is due to increased bone resorption in both females and males and is associated with estrogen deficiency. Estrogens inhibit bone turnover by reducing osteoclast-mediated bone resorption and enhancing osteoblast-mediated bone formation. Both estrogen and raloxifene are currently prescribed for the prevention of postmenopausal bone loss, and the recent WHI study indicated that HRT reduces the incidence of osteoporotic fracture in postmenopausal women (36).

Although numerous studies have investigated a possible association between ER $\alpha$ and ER $\beta$ SNPs (including the ESR1 PvuII and $X b a I$ polymorphisms) and osteoporotic risk, this association remains controversial and requires further investigation (53). A role for the ER in development of osteopenia and in skeletal growth and maintenance is exemplified by the case of an estrogen-resistant male patient carrying a loss-of-function mutation in the gene encoding $E R \alpha$ (54). This patient demonstrated incomplete epiphyseal closure and reduced bone mineral density (BMD). Patients with a mutation in the gene encoding aromatase also show similar skeletal abnormalities due to the deficiency in estrogen production (55). ER $\alpha$ and ER $\beta$ have been detected in cultured human osteoblast-like cells, and in uncultured osteoblast cells (56-62), osteoclasts $(56-58,63,64)$, and bone marrow cells (65). These studies also indicate that the presence of the receptor varies with age and bone type, i.e., whether the bone is cortical (solid bone) or cancellous (spongy). Interestingly, a 46-kDa isoform of ER $\alpha$ is expressed in human primary osteoblasts and is reported to inhibit proliferation induced by the full-length ER $\alpha$ isoform in a human osteosarcoma cell line (66). The expression of these $2 \mathrm{ER} \alpha$ isoforms may help explain the differential response of bone and other tissues to SERMs.
A role for the ERs in bone has been shown in both cell culture and rodent model systems. In 1988, Komm et al. demonstrated ER expression and estradiol-mediated induction of type I procollagen and TGF- $\beta$ in human clonal osteoblast-like osteosarcoma cells (62). In cultured neonatal rat bone marrow, raloxifene reduces the generation of osteoclasts in response to 1,25-dihydroxyvitamin $\mathrm{D}_{3}$ treatment in a dose-dependent manner and increases the proliferation of primary neonatal mouse osteoblasts (67), suggesting that raloxifene reduces bone turnover by inhibiting bone resorption and promoting bone formation. In rodents, osteoblasts and osteoclasts also express ERs, and studies indicate an age- and sex-specific effect of estrogens on bone (68). To date, data from ER-null mouse models have yielded equivocal results (68). Femur length (FL) and BMD are used as measures of rodent bone growth, development, and maintenance. Several studies report reduced FL in both female and male adult mice lacking ER $\alpha$ compared with wild-type animals (69-71), although other studies report no change (72). The data regarding FL in ER $\beta$-null mice are also conflicting and require further study. BMD has also been investigated in ER knockout animals. However, contrary reports exist with respect to the effect of the ER on adult BMD, and this effect varies with ER isoform, type of bone (cancellous or cortical), age, and sex (71-73).

Both treatment with estradiol and effect of gonadectomy have also been used as measures of estrogen's effect on bone in ER knockout animals. Gonadectomy induces bone loss in both sexes, and neither $\alpha E R K O$ nor $\beta E R K O$ mice are protected against cancellous bone loss after ovariectomy $(68,73)$. Pharmacological doses of estradiol stimulate cancellous bone formation in intact female mice, and this effect is blocked by the ER antagonist ICI 182,780, suggesting a role for ER in this process (74). This hypothesis is supported by studies in which estradiol stimulates cancellous bone formation in male wild-type mice, but not in male $\alpha$ ERKO mice (75). Taken together, these findings suggest that ER $\alpha$ mediates estrogen-stimulated net increase in bone formation, while ER $\beta$ 's role in bone is less clear.

\section{Neurodegenerative diseases}

There are clinical and experimental data to support a protective effect of estrogens against neurodegenerative disease in humans. Several recent reviews described the various mechanisms by which estrogens may provide neuroprotection (76-79). Below, we focus on data illustrating estrogen's neuroprotective effects in stroke, Parkinson disease (PD), and Alzheimer disease (AD).

Stroke. Premenopausal women exhibit a lower risk of stroke compared with men; however, this difference is not observed when postmenopausal women are compared with men, suggesting that estrogen protects against stroke. The ESR1 PvuII polymorphism is associated with a higher risk of stroke in men (80). Several observational studies also report an association between estrogen therapy and a lower risk for cardiovascular disease (81). Still, the efficacy of postmenopausal estrogen therapy or HRT in reducing the incidence or recurrence of stroke remains controversial $(36,82-84)$. In the recent Heart and Estrogen-Progestin Replacement Study (HERS) trial, the risk of stroke in women with known coronary artery disease was not reduced by HRT treatment (85). This conclusion was supported by results from both arms of the recent WHI study (estrogen therapy alone, and estrogen combined with progestin [HRT]). Neither HRT nor estrogen therapy reduced the risk of stroke in the WHI study; in fact, an increased risk of stroke was reported in both arms $(36,82)$. There are probably several reasons for this apparent lack of agreement between experimental and 
observational studies and the recent randomized control trials (81); however, one of the key issues currently under discussion is the time from menopause at which hormone treatment is initiated (the "timing hypothesis," which has recently been validated in a prospective analysis of data from the Nurses' Health Study) $(86,87)$. This hypothesis suggests that a window of opportunity exists at perimenopause during which hormone therapy is beneficial to protect against cardiovascular disease, but that these beneficial effects are lost if treatment is begun later in the postmenopausal years. Although the majority of women in observational studies are perimenopausal upon initiation of hormone therapy, recent randomized clinical trials have studied older women who have been postmenopausal for varying numbers of years upon initiation of treatment. The Heart and Estrogen-Progestin Replacement Study (HERS) trial involved older women (beyond 65 years), and women in both arms of the WHI trials also were well into menopause upon treatment. Primate models indicate that HRT must be administered soon after estrogen deficiency to inhibit progression of coronary artery atherosclerosis, and that protection is lost when hormone treatment is delayed for 2 years (comparable to 6 years in women) (88). Therefore, more studies involving perimenopausal women will be required to further test this hypothesis, and to reduce the divergence between experimental data, observational studies, and results from randomized control trials. Development of new experimental models may also contribute to this understanding.

Experimental evidence from animal and cell culture models show that estrogen treatment protects against neuronal cell death due to insult (78). Experimental data also suggest that estrogens protect against stroke. Both ER $\alpha$ and $E R \beta$ are found in various regions of the human and rodent brain, including the hypothalamus, hippocampus, cerebral cortex, midbrain, brainstem, and forebrain, and ER-mediated effects are thought to provide neuroprotection. In an ovariectomized/ischemia mouse model of stroke, estradiol treatment protects wild-type and ER $\beta$-null mice from brain injury, while this protection is abolished in ER $\alpha$-null animals (89), suggesting an important role for ER $\alpha$. Still, a separate study in which stroke is induced by reversible middle cerebral artery occlusion found no increased tissue damage in ER $\alpha$-null females, suggesting that non-ER events are involved (90). A recent explanation for estrogen-mediated neuroprotection is an ER-dependent increase in the angiogenic factor angiopoietin- 1 in the brains of ovariectomized rats treated with estradiol, an effect that is lost in $\alpha$ ERKO mice (91). Therefore, this protection by estrogen may involve both ER-dependent and ER-independent mechanisms.

Parkinson disease and Alzheimer disease. PD results from the degeneration of dopamine-synthesizing (DA-synthesizing) neurons in the substantia nigra, leading to a corresponding reduction of DA levels in the striatum, where the terminals of these neurons are located. The cause of cell death remains unknown, but PD is certainly more prevalent in men than in women. In addition, experimental studies suggest that estrogens may influence the onset and severity of PD. The clinical link between estrogen and PD remains controversial, with studies indicating either an increase or a decrease in disease severity after estrogen therapy, or no effect at all (92). Some reports indicate that postmenopausal estrogen therapy can reduce the risk of PD (93).

Cell culture and animal studies indicate that estrogens regulate dopaminergic neurotransmission by influencing DA synthesis, uptake, and release (94). Estrogen may inhibit dopaminergic degeneration by a variety of mechanisms, including reducing apoptosis, regulating neurotrophic growth factors, reducing neuroinflammation, regulating $\mathrm{NO}$ levels in brain vasculature, and protecting against damage from oxidative stress $(78,94)$. In rodent models of PD, endogenous estradiol in female rats or exogenous estradiol administration after ovariectomy protects against loss of DA content and cell death after insult $(95,96)$. Cell culture studies indicate that ERs may mediate some of these effects. ER $\alpha$ and ER $\beta$ transcripts and protein are reported in the rodent substantia nigra (97-99). Rat mesencephalic neurons cultured in estradiolcontaining medium are resistant to apoptosis and neuronal injury, and this effect is blocked by ICI 182,780, suggesting a role for ER in this protection (100-102). Interestingly, tamoxifen did not block the effect of estrogen in these studies, but rather exaggerated neurotoxicity when given alone $(100,101)$. Further clinical and experimental research will be required to determine whether SERMs or estrogen therapy will be a useful therapeutic approach for ameliorating the severity or incidence of PD.

$\mathrm{AD}$ is the primary cause of dementia in old age and is characterized by a loss of memory and other cognitive abilities. Although numerous case-control and cohort studies (103) indicate that estrogen therapy reduces the risk of $\mathrm{AD}$ in women, data from randomized controlled treatment studies indicate that estrogen therapy is not an effective treatment for postmenopausal women already diagnosed with $\mathrm{AD}$ (103). In the Women's Health Initiative Memory Study (WHIMS), conducted in association with the WHI study, dementia increased in response to both estrogen therapy and HRT, again in contrast to previous indications from observational studies that hormone treatment reduces the risk of $\mathrm{AD}$ (104). As previously mentioned for cardiovascular disease, it has been suggested that estrogen treatment in early menopause (rather than later in menopause) might delay the onset of age-related cognitive changes or reduce the risk or severity of AD (104).

$\mathrm{AD}$ is characterized histopathologically by the presence of neurofibrillary tangles and neuritic plaques, whose major components include hyperphosphorylated tau protein and $\beta$-amyloid protein, respectively (104). Accumulation of these proteins is thought to eventually result in neural cell death. Estrogens may reduce the risk or severity of $\mathrm{AD}$ via reducing oxidative stress, increasing cerebral blood flow, and increasing glucose transport (104). In addition to its effect on the dopaminergic system, estrogen also affects the cholinergic, serotonergic, and noradrenergic neurotransmitter systems. In an ovariectomized rat model, estrogen treatment increases choline acetyltransferase activity, which regulates the synthesis of acetylcholine, a neurotransmitter that is known to be present at reduced levels in AD (105). Estradiol treatment of ovariectomized guinea pigs reduces the amount of $\beta$-amyloid protein in brain extracts (106). Furthermore, estradiol reduces $\beta$-amyloid-induced cell death in a cholinergic cell line, and this effect is blocked by ICI 182,780 , suggesting an ER-mediated mechanism (107). Another hallmark of $\mathrm{AD}$ is a loss of synapses and dendritic branches, and estrogens have been shown to affect these structures (104). Therefore, while experimental evidence suggests that estrogens may be beneficial in reducing the risk of $\mathrm{AD}$, further clinical investigation in pre/perimenopausal and postmenopausal women is needed.

\section{Cardiovascular disease}

The incidence of cardiovascular diseases is low in premenopausal women but increases substantially after menopause (matching that seen in men), suggesting that estrogens protect the female cardiovascular system. Although for years HRT was prescribed to 
postmenopausal women to provide protection from cardiovascular disease, a summary of recent clinical trials indicates that such treatment may have had negative cardiovascular consequences namely, an increased risk of venous thromboembolism or stroke (108). As previously mentioned (see "Stroke," above), the discrepancy between observational and recent randomized control studies may be related to the age at treatment initiation - the "timing hypothesis." SERMs may be useful for the reduction of cardiovascular risk, and in the current Raloxifene Use for the Heart (RUTH) study, the efficacy of raloxifene treatment in lowering the risk of cardiovascular disease in women is being investigated (8).

Reduced levels of ER $\alpha$ have been associated with the development of coronary artery disease in females (109). In coronary artery samples from pre- and postmenopausal women, ER $\alpha$ was expressed in the majority of normal arteries, but only in a minority of arteries obtained from women with coronary artery disease (109). This is supported by evidence indicating an increase in methylation (and an associated decrease in expression) of the gene encoding ER $\alpha$ in human coronary atherosclerotic plaques compared with normal aortic tissue (110).

Polymorphisms in both ERs have been associated with cardiovascular disease. Polymorphisms in ER $\alpha$ that link it to severity and risk of coronary artery disease have been identified (111-113). In the Framingham Heart Study, the ESR1 PvuII polymorphism is associated with an increased risk of myocardial infarction in men (114), and with blood pressure variation in men (115). In the Victorian Family Heart Study, this polymorphism was also associated with increased systolic blood pressure in men (116). In addition, the haplotype encompassing both ESR1 PvuII and XbaI polymorphisms is associated with an increased risk of myocardial infarction and ischemic heart disease in postmenopausal women in the Rotterdam Study (117). ER $\beta$ polymorphisms have also been associated with left ventricular mass and left ventricular wall thickness in women with hypertension (118), and with blood pressure in men (116) and in healthy postmenopausal Japanese women (119). These associations are supported by data from animal models indicating that mice lacking ER $\beta$ develop hypertension upon aging and exhibit abnormal vascular function (120).

Data from animal model and clinical studies also support a protective role for estrogens in the regulation of lipid and cholesterol levels, direct effects on vascular cells, and recovery from vascular injury. The ERs are thought to be involved in this protective effect. Estrogen is known to increase HDL plasma levels; however, this response varies greatly in women and may be due in part to genetic factors. ESR1 polymorphisms are associated with increased HDL levels in postmenopausal women with coronary disease in response to estrogen therapy or HRT (121). In addition, an ESR2 polymorphism is associated with reduced LDL cholesterol levels in premenopausal or postmenopausal women exposed to HRT, but not in postmenopausal women unexposed to HRT (122). Estrogens induce several effects in the vasculature, including vasodilation, which is considered a rapid nongenomic effect because it occurs 5-20 minutes after estrogen exposure and does not require changes in gene expression. Genomic effects of estrogens on blood vessels include inhibition of the injury response and protection against atherosclerosis (123). ER $\alpha$, but not ER $\beta$, mediates estrogen's regulation of serum lipid and cholesterol concentrations in mice (124). Mice carrying a disruption of the Apoe gene (Apoe $e^{-/-}$mice) develop spontaneous atherosclerotic lesions and possess elevated plasma cholesterol levels similar to those seen clinically (125), but these phenotypes are reduced by treatment with estra- diol (126, S1). When Apoe $e^{-/}$mice are crossed with ER $\alpha$-null mice and treated with estradiol, the protective effect of estradiol is lost in terms of both reduced cholesterol levels and lesion size (S2). Interestingly, estradiol still reduces the histological complexity of the atherosclerotic plaques in the compound gene-targeted animals, suggesting that ER $\alpha$ may be involved in some elements of plaque formation.

Although the cardioprotective effects of estrogens can be attributed in part to indirect actions via a reduction in circulating lipid levels, a direct effect on blood vessels has also been demonstrated, and evidence suggests that this effect may occur via ERs. Blood vessel walls consist of smooth muscle cells and an endothelial cell lining. In humans, both ER $\alpha$ and ER $\beta$ are expressed in vascular endothelial cells, vascular smooth muscle cells, and cardiomyocytes $(87,123)$. NO released by vascular endothelial cells induces relaxation of vascular smooth muscle and inhibits activation of platelets. Estrogen triggers the rapid release of NO from cultured endothelial cells by a nongenomic mechanism, which involves activation of eNOS through an ER $\alpha$-dependent mechanism in cultured ovine endothelial cells (S3). In addition to activating eNOS, estradiol increases the abundance of eNOS by activating transcription of the eNOS gene through an ER-dependent mechanism (S4). The in vitro data supporting a role for the ER in regulating the levels of active eNOS are consistent with premature coronary artery disease in the male patient with an ER mutation. The patient lacks flowmediated, endothelium-dependent peripheral vasodilation, which is attributed to a lack of NO production (S5, S6). A similar reduction in NO production is observed in male $\alpha$ ERKO animals (S7).

Estrogens protect vascular endothelial cells against injury in a mouse carotid injury model, and this protection is maintained in ER $\beta$-null mice (S8) but lost in ER $\alpha$-null mice (S9), indicating that ER $\alpha$ is required for this protection. Some studies support a role for the individual receptors in mediating estrogen's protective effects on vascular injury. In a different carotid injury model, estradiol treatment enhances reendothelialization (endothelial cell growth) after injury in ovariectomized wild-type and ER $\beta$-null animals, but this effect is lost in ER $\alpha$-null animals (S10). In a similar study with ER knockout animals, ER $\alpha$ is required for cardioprotection in a myocardial ischemia model (S11). After myocardial infarction, $\beta E R K O$ animals demonstrate increased mortality and incidence of markers of heart failure, suggesting that ER $\beta$ attenuates the development of chronic heart failure (S12). Similarly, recent experimental studies reflective of a pressure overload left ventricular hypertrophy indicate that $\operatorname{ER} \beta$ mediates the protective effect of estrogen in female mice (S13).

\section{Obesity}

Obesity results from excess white adipose tissue, which is considered to be an endocrine organ based on its ability to store and metabolize steroid hormones. Estrogens regulate both the metabolism and the location of white adipose tissue and play a role in adipogenesis, adipose deposition, lipogenesis, lipolysis, and adipocyte proliferation (S14). In women, the loss of circulating estrogen following menopause is associated with an increase in central body fat, and this effect is attenuated by estrogen treatment (S15).

Many groups have demonstrated that $\operatorname{ER} \alpha$ and ER $\beta$ mRNA and protein are expressed in human adipose tissue (S16-S22). In mature human adipocytes, ER $\alpha$ is the more highly expressed isoform, while ER $\beta$ appears to be more highly expressed in women than in men (S21). Interestingly, only ER $\alpha$, not ER $\beta$, is expressed in preadipocytes, although the expression of $\mathrm{ER} \alpha$ in 
preadipocytes is very low compared with that in mature adipocytes (S21). Both ERs are also expressed in rat adipose tissue, again with a predominance of ER $\alpha$ (S23).

Although the mechanisms responsible for estrogen's regulation of adipogenesis remain unclear (S16), they may involve estrogen's suppression of lipoprotein lipase (LPL), a lipogenic enzyme that regulates the metabolism of plasma triglycerides to free fatty acids and increases lipid storage by adipocytes. The LPL gene is a direct transcriptional target of estrogen (S24). In women, LPL levels increase after menopause (S25). In addition to inhibiting lipogenesis (fatty acid uptake), estrogen also increases lipolysis (fatty acid and glycerol release) by increasing expression of hormone-sensitive lipase, which catalyzes the breakdown of stored triacylglycerol and the release of fatty acids (S26). However, estrogen has also been shown to inhibit lipolysis. Estrogen treatment of postmenopausal women increases expression of the $\alpha_{2 \mathrm{~A}}$-adrenergic receptor in subcutaneous adipose tissue. Activation of this receptor inhibits lipolysis; therefore, increased expression due to estrogen would inhibit lipolysis. This increase was recapitulated in vitro using cultured human tissue adipose fragments incubated with estradiol and was correlated with inhibition of lipolysis (S27), suggesting a direct role for ER in regulating lipolysis. The complex relationship between the ERs and adipogenesis remains to be elucidated and may provide critical information for the prevention and treatment of obesity.

There is also evidence for rapid nongenomic estrogen actions via a membrane-bound ER in adipocytes (S28). Rodent models also support a role for ER in human adipose tissue. Both male and female $\alpha E R K O$ mice, as well as aromatase knockout mice, possess $50-100 \%$ more adipose tissue than do wild-type controls, and this appears to be due to increases in both adipocyte number and size, implicating a role for $\mathrm{ER} \alpha$ in adipocyte growth and proliferation (S29). In addition, a role for ER $\beta$ in the effect of estrogen on adipose tissue has been suggested (S30). Ovariectomy of ER $\alpha$ animals decreases adipose tissue and body weight, suggesting that ER $\beta$ may also be involved in adipogenesis in some undetermined manner (S30).

\section{Concluding remarks}

Loss of estrogen or its receptor(s) contributes to the development or progression of various diseases. Both activation (via estrogen agonists) and inhibition (via estrogen antagonists) of ER action are therapeutic strategies currently used in the clinical setting. ER antagonists are effective for the treatment of breast cancer, and their efficacy in the treatment of other hormone-dependent cancers awaits further study. However, despite supporting evidence from animal models, the utility of estrogen treatment to reduce the risk or severity of neurodegenerative or cardiovascular disease remains unsettled, and studies disagree on the optimum dose and frequency of treatment, as well as the optimum patient age at treatment initiation. Correct timing of treatment initiation during the "window of opportunity" may be required to determine the true efficacy of estrogen treatment. In addition, tailoring the type and duration of hormone therapy based on genetic profile may provide a means to optimize treatment for each patient. Development of tissue-specific SERMs, such as those specific to the brain (S31), vasculature, and skeleton, might alleviate some of the risks associated with traditional estrogen therapy complicated by effects in other tissues. Given that estrogen is the most consistently effective treatment for vasomotor symptoms, development of SERMs specific for these symptoms would be particularly desirable. A role for estrogen and ER $\alpha$ in male fertility has also been demonstrated, and further investigation of these mechanisms may provide novel therapeutic strategies for male infertility (S32). Finally, diseases exist for which estrogen has been implicated in their pathogenesis but a definitive role for the ER has yet to be established. These include endometriosis and polycystic ovary syndrome, and diseases with sex-specific biases, such as lupus erythematosus, which is more common in females than in males. What role the ER may play in the risk or severity of these and other diseases will no doubt increase our ever-expanding knowledge of the relationship among estrogen, ERs, and disease.

\section{Acknowledgments}

This research was supported by the Intramural Research Program of the NIH, National Institute of Environmental Health Sciences.

Note: References S1-S35 are available online with this article; doi:10.1172/JCI27987DS1.

Address correspondence to: Kenneth S. Korach, Receptor Biology Section, Laboratory of Reproductive and Developmental Toxicology, National Institute of Environmental Health Sciences, National Institutes of Health, Maryland B3-02, PO Box 12233, Research Triangle Park, North Carolina 27709, USA. Phone: (919) 541-3512; Fax: (919) 541-0696; E-mail: korach@niehs.nih.gov.
1. Mueller, S.O., and Korach, K.S. 2001. Estrogen receptors and endocrine diseases: lessons from estrogen receptor knockout mice. Curr. Opin. Pharmacol. 1:613-619.

2. Couse, J.F., and Korach, K.S. 1999. Estrogen receptor null mice: what have we learned and where will they lead us? Endocr. Rev. 20:358-417.

3. Herynk, M.H., and Fuqua, S.A. 2004. Estrogen receptor mutations in human disease. Endocr. Rev. 25:869-898.

4. Fabian, C.J., and Kimler, B.F. 2005. Selective estrogen-receptor modulators for primary prevention of breast cancer. J. Clin. Oncol. 23:1644-1655.

5. Shang, Y., and Brown, M. 2002. Molecular determinants for the tissue specificity of SERMs. Science. 295:2465-2468.

6. Henderson, B.E., and Feigelson, H.S. 2000. Hormonal carcinogenesis. Carcinogenesis. 21:427-433.

7. Yue, W., et al. 2005. Tamoxifen versus aromatase inhibitors for breast cancer prevention. Clin. Cancer Res. 11:925s-930s.

8. Mosca, L. 2001. Rationale and overview of the Ral- oxifene Use for the Heart (RUTH) trial. Ann. N. Y. Acad. Sci. 949:181-185.

9. Zuppan, P.J., et al. 1989. Polymorphisms at the estrogen receptor (ESR) locus and linkage relationships on chromosome 6q [abstract]. Cytogenet. Cell Genet. 51:1116.

10. Yaich, L., Dupont, W.D., Cavener, D.R., and Parl, F.F. 1992. Analysis of the PvuII restriction fragment-length polymorphism and exon structure of the estrogen receptor gene in breast cancer and peripheral blood. Cancer Res. 52:77-83.

11. Cai, Q., et al. 2003. Genetic polymorphisms in the estrogen receptor alpha gene and risk of breast cancer: results from the Shanghai Breast Cancer Study. Cancer Epidemiol. Biomarkers Prev. 12:853-859.

12. Zheng, S.L., et al. 2003. Joint effect of estrogen receptor beta sequence variants and endogenous estrogen exposure on breast cancer risk in Chinese women. Cancer Res. 63:7624-7629.

13. Maguire, P., et al. 2005. Estrogen receptor Beta (ESR2) polymorphisms in familial and sporadic breast cancer. Breast Cancer Res. Treat. 94:145-152.
14. Bocchinfuso, W.P., and Korach, K.S. 1997. Mammary gland development and tumorigenesis in estrogen receptor knockout mice. J. Mammary Gland Biol. Neoplasia. 2:323-334.

15. Hewitt, S.C., et al. 2002. Lack of ductal development in the absence of functional estrogen receptor alpha delays mammary tumor formation induced by transgenic expression of ErbB2/neu. Cancer Res. 62:2798-2805

16. Yoshidome, K., Shibata, M.A., Couldrey, C., Korach, K.S., and Green, J.E. 2000. Estrogen promotes mammary tumor development in C3(1)/SV40 large $\mathrm{T}$-antigen transgenic mice: paradoxical loss of estrogen receptoralpha expression during tumor progression. Cancer Res. 60:6901-6910.

17. Xu, J., and Li, Q. 2003. Review of the in vivo functions of the 160 steroid receptor coactivator family. Mol. Endocrinol. 17:1681-1692.

18. Liao, L., et al. 2002. Molecular structure and biological function of the cancer-amplified nuclear receptor coactivator SRC-3/AIB1. J. Steroid Biochem. Mol. Biol. 83:3-14. 
19. Edwards, B.K., et al. 2005. Annual report to the nation on the status of cancer, 1975-2002, featuring population-based trends in cancer treatment. J. Natl. Cancer Inst. 97:1407-1427.

20. Chu, S., Mamers, P., Burger, H.G., and Fuller, P.J. 2000. Estrogen receptor isoform gene expression in ovarian stromal and epithelial tumors. J. Clin. Endocrinol. Metab. 85:1200-1205.

21. Syed, V., Ulinski, G., Mok, S.C., Yiu, G.K., and Ho, S.M. 2001. Expression of gonadotropin receptor and growth responses to key reproductive hormones in normal and malignant human ovarian surface epithelial cells. Cancer Res. 61:6768-6776.

22. Nash, J.D., Ozols, R.F., Smyth, J.F., and Hamilton, T.C. 1989. Estrogen and anti-estrogen effects on the growth of human epithelial ovarian cancer in vitro. Obstet. Gynecol. 73:1009-1016.

23. Jager, W., et al. 1995. A randomized comparison of triptorelin and tamoxifen as treatment of progressive ovarian cancer. Anticancer Res. 15:2639-2642.

24. Hatch, K.D., Beecham, J.B., Blessing, J.A., and Creasman, W.T. 1991. Responsiveness of patients with advanced ovarian carcinoma to tamoxifen. A Gynecologic Oncology Group study of second-line therapy in 105 patients. Cancer. 68:269-271.

25. Risma, K.A., et al. 1995. Targeted overexpression of luteinizing hormone in transgenic mice leads to infertility, polycystic ovaries, and ovarian tumors. Proc. Natl. Acad. Sci. U. S. A. 92:1322-1326.

26. Couse, J.F., et al. 2004. Formation of cystic ovarian follicles associated with elevated luteinizing hormone requires estrogen receptor-beta. Endocrinology. 145:4693-4702.

27. Burns, K.H., et al. 2003. Sexually dimorphic roles of steroid hormone receptor signaling in gonadal tumorigenesis. Mol. Endocrinol. 17:2039-2052.

28. Arai, N., Strom, A., Rafter, J.J., and Gustafsson, J.A. 2000. Estrogen receptor beta mRNA in colon cancer cells: growth effects of estrogen and genistein. Biochem. Biophys. Res. Commun. 270:425-431.

29. Fiorelli, G., Picariello, L., Martineti, V., Tonelli, F. and Brandi, M.L. 1999. Functional estrogen receptor beta in colon cancer cells. Biochem. Biophys. Res. Commun. 261:521-527.

30. Foley, E.F., Jazaeri, A.A., Shupnik, M.A., Jazaeri, O., and Rice, L.W. 2000. Selective loss of estrogen receptor beta in malignant human colon. Cancer Res. 60:245-248.

31. Qiu, Y., Waters, C.E., Lewis, A.E., Langman, M.J., and Eggo, M.C. 2002. Oestrogen-induced apoptosis in colonocytes expressing oestrogen receptor beta. J. Endocrinol. 174:369-377.

32. Jassam, N., Bell, S.M., Speirs, V., and Quirke, P. 2005. Loss of expression of oestrogen receptor beta in colon cancer and its association with Dukes' staging. Oncol. Rep. 14:17-21.

33. Konstantinopoulos, P.A., et al. 2003. Oestrogen receptor beta (ERbeta) is abundantly expressed in normal colonic mucosa, but declines in colon adenocarcinoma paralleling the tumour's dedifferentiation. Eur. J. Cancer. 39:1251-1258.

34. Campbell-Thompson, M., Lynch, I.J., and Bhardwaj, B. 2001. Expression of estrogen receptor (ER) subtypes and ERbeta isoforms in colon cancer. Cancer Res. 61:632-640.

35. Jemal, A., et al. 2004. Cancer statistics, 2004. CA Cancer J. Clin. 54:8-29.

36. Rossouw, J.E., et al. 2002. Risks and benefits of estrogen plus progestin in healthy postmenopausal women: principal results from the Women's Health Initiative randomized controlled trial. JAMA 288:321-333.

37. Harris, H.A., et al. 2003. Evaluation of an estrogen receptor-beta agonist in animal models of human disease. Endocrinology. 144:4241-4249.

38. Picariello, L., et al. 2003. Growth response of colon cancer cell lines to selective estrogen receptor modulators. Anticancer Res. 23:2419-2424.
39. Bosland, M.C. 2000. The role of steroid hormones in prostate carcinogenesis. J. Natl. Cancer Inst. Monogr. 27:39-66

40. Harkonen, P.L., and Makela, S.I. 2004. Role of estrogens in development of prostate cancer. J. Steroid Biochem. Mol. Biol. 92:297-305.

41. Leav, I., et al. 2001. Comparative studies of the estrogen receptors beta and alpha and the androgen receptor in normal human prostate glands, dysplasia, and in primary and metastatic carcinoma. Am. J. Pathol. 159:79-92.

42. Horvath, L.G., et al. 2001. Frequent loss of estrogen receptor-beta expression in prostate cancer. Cancer Res. 61:5331-5335.

43. Imamov, O., et al. 2004. Estrogen receptor beta regulates epithelial cellular differentiation in the mouse ventral prostate. Proc. Natl. Acad. Sci. U. S. A 101:9375-9380.

44. Krishnan, G., et al. 2004. Novel ERbeta selective agonists induce prostate atrophy in rodents without affecting the hypothalamo-pituitary-gonadal axis. In The Endocrine Society Annual Meeting 2004. The Endocrine Society Press. Chevy Chase, Maryland, USA. $180-181$

45. Huang, L., Pu, Y., Alam, S., Birch, L., and Prins, G.S. 2004. Estrogenic regulation of signaling pathways and homeobox genes during rat prostate development. J. Androl. 25:330-337.

46. Prins, G.S., et al. 2001. Estrogen imprinting of the developing prostate gland is mediated through stromal estrogen receptor alpha: studies with alphaERKO and betaERKO mice. Cancer Res. 61:6089-6097.

47. Risbridger, G., et al. 2001. Evidence that epithelial and mesenchymal estrogen receptor-alpha mediates effects of estrogen on prostatic epithelium. Dev. Biol. 229:432-442.

48. Bergan, R.C., et al. 1999. A Phase II study of highdose tamoxifen in patients with hormone-refractory prostate cancer. Clin. Cancer Res. 5:2366-2373.

49. Stein, S., et al. 2001. Phase II trial of toremifene in androgen-independent prostate cancer: a Penn cancer clinical trials group trial. Am. J. Clin. Oncol. 24:283-285.

50. Lax, S.F. 2004. Molecular genetic pathways in various types of endometrial carcinoma: from a phenotypical to a molecular-based classification. Virchows Arch. 444:213-223.

51. Grady, D., Gebretsadik, T., Kerlikowske, K., Ernster, V., and Petitti, D. 1995. Hormone replacement therapy and endometrial cancer risk: a meta-analysis. Obstet. Gynecol. 85:304-313.

52. Wu, H., et al. 2005. Hypomethylation-linked activation of PAX2 mediates tamoxifen-stimulated endometrial carcinogenesis. Nature. 438:981-987.

53. Gennari, L., et al. 2005. Estrogen receptor gene polymorphisms and the genetics of osteoporosis: a HuGE review. Am. J. Epidemiol. 161:307-320.

54. Smith, E.P., et al. 1994. Estrogen resistance caused by a mutation in the estrogen-receptor gene in a man. N. Engl. J. Med. 331:1056-1061.

55. Herrmann, B.L., et al. 2002. Impact of estrogen replacement therapy in a male with congenital aromatase deficiency caused by a novel mutation in the CYP19 gene. J. Clin. Endocrinol. Metab. 87:5476-5484.

56. Batra, G.S., et al. 2003. Evidence for cell-specific changes with age in expression of oestrogen receptor (ER) alpha and beta in bone fractures from men and women. J. Pathol. 200:65-73.

57. Braidman, I.P., et al. 2001. Localization of estrogen receptor beta protein expression in adult human bone. J. Bone Miner. Res. 16:214-220.

58. Bord, S., Horner, A., Beavan, S., and Compston, J. 2001. Estrogen receptors alpha and beta are differentially expressed in developing human bone. J. Clin. Endocrinol. Metab. 86:2309-2314.

59. Stulc, T., Klement, D., Kvasnicka, J., and Stepan, J.J. 1997. Immunocytochemical detection of estro- gen receptors in bone cells using flow cytometry. Biochim. Biophys. Acta. 1356:95-100.

60. Vidal, O., Kindblom, L.G., and Ohlsson, C. 1999. Expression and localization of estrogen receptorbeta in murine and human bone. J. Bone Miner. Res. 14:923-929.

61. Eriksen, E.F., et al. 1988. Evidence of estrogen receptors in normal human osteoblast-like cells. Science. 241:84-86.

62. Komm, B.S., et al. 1988. Estrogen binding, receptor mRNA, and biologic response in osteoblast-like osteosarcoma cells. Science. 241:81-84

63. Pensler, J.M., Radosevich, J.A., Higbee, R., and Langman, C.B. 1990. Osteoclasts isolated from membranous bone in children exhibit nuclear estrogen and progesterone receptors. J. Bone Miner. Res. 5:797-802.

64. Oreffo, R.O., et al. 1999. Expression of estrogen receptor-alpha in cells of the osteoclastic lineage. Histochem. Cell Biol. 111:125-133.

65. Bord, S., Vedi, S., Beavan, S.R., Horner, A., and Compston, J.E. 2000. Megakaryocyte population in human bone marrow increases with estrogen treatment: a role in bone remodeling? Bone. 27:397-401.

66. Denger, S., et al. 2001. ERalpha gene expression in human primary osteoblasts: evidence for the expression of two receptor proteins. Mol. Endocrinol. 15:2064-2077.

67. Taranta, A., et al. 2002. The selective estrogen receptor modulator raloxifene regulates osteoclast and osteoblast activity in vitro. Bone. 30:368-376.

68. Windahl, S.H., Andersson, G., and Gustafsson, J.A. 2002. Elucidation of estrogen receptor function in bone with the use of mouse models. Trends Endocrinol. Metab. 13:195-200.

69. Vidal, O., et al. 1999. Disproportional body growth in female estrogen receptor-alpha-inactivated mice. Biochem. Biophys. Res. Commun. 265:569-571.

70. Vidal, O., et al. 2000. Estrogen receptor specificity in the regulation of skeletal growth and maturation in male mice. Proc. Natl. Acad. Sci. U. S. A. 97:5474-5479.

71. Lindberg, M.K., et al. 2001. Estrogen receptor specificity in the regulation of the skeleton in female mice. J. Endocrinol. 171:229-236.

72. Sims, N.A., et al. 2002. Deletion of estrogen receptors reveals a regulatory role for estrogen receptors-beta in bone remodeling in females but not in males. Bone. 30:18-25

73. Windahl, S.H., Vidal, O., Andersson, G., Gustafsson, J.A., and Ohlsson, C. 1999. Increased cortical bone mineral content but unchanged trabecular bone mineral density in female $\operatorname{ER} \beta(-/-)$ mice. J. Clin. Invest. 104:895-901.

74. Samuels, A., Perry, M.J., Goodship, A.E., Fraser, W.D., and Tobias, J.H. 2000. Is high-dose estrogeninduced osteogenesis in the mouse mediated by an estrogen receptor? Bone. 27:41-46.

75. McDougall, K.E., et al. 2003. Estrogen receptor-alpha dependency of estrogen's stimulatory action on cancellous bone formation in male mice. Endocrinology. 144:1994-1999.

76. Garcia-Segura, L.M., Azcoitia, I., and DonCarlos, L.L. 2001. Neuroprotection by estradiol. Prog. Neurobiol. 63:29-60.

77. Wise, P.M., Dubal, D.B., Wilson, M.E., Rau, S.W., and Bottner, M. 2001. Minireview. Neuroprotective effects of estrogen: new insights into mechanisms of action. Endocrinology. 142:969-973.

78. Amantea, D., Russo, R., Bagetta, G., and Corasaniti, M.T. 2005. From clinical evidence to molecular mechanisms underlying neuroprotection afforded by estrogens. Pharmacol. Res. 52:119-132.

79. Kajta, M., and Beyer, C. 2003. Cellular strategies of estrogen-mediated neuroprotection during brain development [review]. Endocrine. 21:3-9.

80. Shearman, A.M., et al. 2005. Estrogen receptor 
alpha gene variation and the risk of stroke. Stroke. 36:2281-2282.

81. Grodstein, F., Clarkson, T.B., and Manson, J.E. 2003. Understanding the divergent data on postmenopausal hormone therapy. N. Engl. J. Med. 348:645-650.

82. Rossouw, J.E. 2005. Coronary heart disease in menopausal women: implications of primary and secondary prevention trials of hormones. Maturitas. 51:51-63.

83. Hurn, P.D., and Sacco, R.L. 2004. Sex, steroids, and stroke: introduction. Stroke. 35:2642-2643.

84. Brass, L.M. 2004. Hormone replacement therapy and stroke: clinical trials review. Stroke. 35:2644-2647.

85. Simon, J.A., et al. 2001. Postmenopausal hormone therapy and risk of stroke: the Heart and Estrogenprogestin Replacement Study (HERS). Circulation 103:638-642.

86. Grodstein, F., Manson, J.E., and Stampfer, M.J. 2006. Hormone therapy and coronary heart disease: the role of time since menopause and age at hormone initiation. J. Womens Health (Larchmt.). 15:35-44.

87. Mendelsohn, M.E., and Karas, R.H. 2005. Molecular and cellular basis of cardiovascular gender differences. Science. 308:1583-1587.

88. Clarkson, T.B., and Appt, S.E. 2005. Controversies about HRT: lessons from monkey models. Maturitas. 51:64-74.

89. Dubal, D.B., et al. 2001. Estrogen receptor alpha, not beta, is a critical link in estradiol-mediated protection against brain injury. Proc. Natl. Acad. Sci.U.S. A. 98:1952-1957.

90. Sampei, K., et al. 2000. Stroke in estrogen receptoralpha-deficient mice. Stroke. 31:738-743; discussion 744

91. Ardelt, A.A., et al. 2005. Estradiol regulates angiopoietin-1 mRNA expression through estrogen receptor-alpha in a rodent experimental stroke model. Stroke. 36:337-341.

92. Saunders-Pullman, R. 2003. Estrogens and Parkinson disease: neuroprotective, symptomatic, neither, or both? Endocrine. 21:81-87.

93. Currie, L.J., Harrison, M.B., Trugman, J.M., Bennett, J.P., and Wooten, G.F. 2004. Postmenopausal estrogen use affects risk for Parkinson disease. Arch. Neurol. 61:886-888.

94. Shulman, L.M. 2002. Is there a connection between estrogen and Parkinson's disease? Parkinsonism Relat. Disord. 8:289-295.

95. Gillies, G.E., Murray, H.E., Dexter, D., and McArthur, S. 2004. Sex dimorphisms in the neuroprotective effects of estrogen in an animal model of Parkinson's disease. Pharmacol. Biochem. Behav. 78:513-522.

96. Quesada, A., and Micevych, P.E. 2004. Estrogen interacts with the IGF-1 system to protect nigrostriatal dopamine and maintain motoric behavior after 6-hydroxdopamine lesions. J. Neurosci. Res. 75:107-116.
97. Ravizza, T., Galanopoulou, A.S., Veliskova, J., and Moshe, S.L. 2002. Sex differences in androgen and estrogen receptor expression in rat substantia nigra during development: an immunohistochemical study. Neuroscience. 115:685-696.

98. Mitra, S.W., et al. 2003. Immunolocalization of estrogen receptor beta in the mouse brain: comparison with estrogen receptor alpha. Endocrinology. 144:2055-2067.

99. Laflamme, N., Nappi, R.E., Drolet, G., Labrie, C., and Rivest, S. 1998. Expression and neuropeptidergic characterization of estrogen receptors (ERalpha and ERbeta) throughout the rat brain: anatomical evidence of distinct roles of each subtype. J. Neurobiol. 36:357-378.

100.Sawada, H., et al. 1998. Estradiol protects mesencephalic dopaminergic neurons from oxidative stress-induced neuronal death. J. Neurosci. Res. 54:707-719.

101.Sawada, H., et al. 2000. Mechanisms of antiapoptotic effects of estrogens in nigral dopaminergic neurons. FASEB J. 14:1202-1214.

102.Liu, X., et al. 2005. Estrogen provides neuroprotection against activated microglia-induced dopaminergic neuronal injury through both estrogen receptor-alpha and estrogen receptor-beta in microglia. J. Neurosci. Res. 81:653-665.

103. Sherwin, B.B. 2003. Estrogen and cognitive functioning in women. Endocr. Rev. 24:133-151.

104.Pinkerton, J.V., and Henderson, V.W. 2005. Estrogen and cognition, with a focus on Alzheimer's disease. Semin. Reprod. Med. 23:172-179.

105.Luine, V.N. 1985. Estradiol increases choline acetyltransferase activity in specific basal forebrain nuclei and projection areas of female rats. Exp. Neurol. 89:484-490.

106.Petanceska, S.S., Nagy, V., Frail, D., and Gandy, S. 2000. Ovariectomy and 17beta-estradiol modulate the levels of Alzheimer's amyloid beta peptides in brain. Exp. Gerontol. 35:1317-1325.

107. Marin, R., et al. 2003. Estradiol prevents amyloidbeta peptide-induced cell death in a cholinergic cell line via modulation of a classical estrogen receptor. Neuroscience. 121:917-926.

108.Farquhar, C.M., Marjoribanks, J., Lethaby, A., Lamberts, Q., and Suckling, J.A. 2005. Long term hormone therapy for perimenopausal and postmenopausal women. Cochrane Database Syst. Rev. 3:CD004143.

109.Losordo, D.W., Kearney, M., Kim, E.A., Jekanowski, J., and Isner, J.M. 1994. Variable expression of the estrogen receptor in normal and atherosclerotic coronary arteries of premenopausal women. Circulation. 89:1501-1510.

110.Post, W.S., et al. 1999. Methylation of the estrogen receptor gene is associated with aging and atherosclerosis in the cardiovascular system. Cardiovasc. Res. 43:985-991.

111.Pollak, A., et al. 2004. Association of oestrogen receptor alpha gene polymorphism with the angio- graphic extent of coronary artery disease. Eur. Heart J. 25:240-245.

112.Kunnas, T.A., Laippala, P., Penttila, A., Lehtimaki, T., and Karhunen, P.J. 2000. Association of polymorphism of human alpha oestrogen receptor gene with coronary artery disease in men: a necropsy study. BMJ. 321:273-274.

113.Lu, H., et al. 2002. Association of estrogen receptoralpha gene polymorphisms with coronary artery disease in patients with familial hypercholesterolemia. Arterioscler. Thromb. Vasc. Biol. 22:817-823.

114.Shearman, A.M., et al. 2003. Association between estrogen receptor alpha gene variation and cardiovascular disease. JAMA. 290:2263-2270.

115.Peter, I., et al. 2005. Variation in estrogen-related genes and cross-sectional and longitudinal blood pressure in the Framingham Heart Study.J. Hypertens. 23:2193-2200.

116.Ellis, J.A., Infantino, T., and Harrap, S.B. 2004. Sex-dependent association of blood pressure with oestrogen receptor genes ERalpha and ERbeta. J. Hypertens. 22:1127-1131.

117. Schuit, S.C., et al. 2004. Estrogen receptor alpha gene polymorphisms and risk of myocardial infarction. JAMA. 291:2969-2977.

118.Peter, I., et al. 2005. Association of estrogen receptor beta gene polymorphisms with left ventricular mass and wall thickness in women. Am. J. Hypertens. 18:1388-1395.

119. Ogawa, S., et al. 2000. Association of estrogen receptor beta (ESR2) gene polymorphism with blood pressure. J. Hum. Genet. 45:327-330.

120.Zhu, Y., et al. 2002. Abnormal vascular function and hypertension in mice deficient in estrogen receptor beta. Science. 295:505-508.

121. Herrington, D.M., et al. 2002. Estrogen-receptor polymorphisms and effects of estrogen replacement on high-density lipoprotein cholesterol in women with coronary disease. N. Engl. J. Med. 346:967-974

122. Almeida, S., et al. 2005. Estrogen receptor 2 and progesterone receptor gene polymorphisms and lipid levels in women with different hormonal status. Pharmacogenomics J. 5:30-34.

123. Mendelsohn, M.E. 2000. Mechanisms of estrogen action in the cardiovascular system. J. Steroid Biochem. Mol. Biol. 74:337-343.

124.Ohlsson, C., et al. 2000. Obesity and disturbed lipoprotein profile in estrogen receptor-alphadeficient male mice. Biochem. Biophys. Res. Commun. 278:640-645.

125.Zhang, S.H., Reddick, R.L., Piedrahita, J.A., and Maeda, N. 1992. Spontaneous hypercholesterolemia and arterial lesions in mice lacking apolipoprotein E. Science. 258:468-471.

126. Bourassa, P.A., Milos, P.M., Gaynor, B.J., Breslow, J.L., and Aiello, R.J. 1996. Estrogen reduces atherosclerotic lesion development in apolipoprotein Edeficient mice. Proc. Natl. Acad. Sci. U. S. A. 93:10022-10027. 